\title{
Nippostrongylinae (Nematoda : Heligmosomidae) néarctiques
}

\author{
par Marie-Claude DURETTE-DESSET \\ Laboratoire de Zoologie (Vers) associé au C.N.R.S. (Pr A.-G. СнаваUD), \\ Muséum national d'Histoire naturelle, 43, rue Cuvier, F 75231 Paris Cedex 05
}

\section{Résumé.}

1) Nous avons tenté d'éclaircir la position systématique de quatre espèces de Nippostrongylinae néarctiques:

- Longistriata carolinensis Dikmans, 1935, parasite de Peromyscus maniculatus, est placé dans le genre Boreostrongylus.

- Boreostrongylus dikmansi n. sp., parasite de Microtus ochrogaster, avait été confondu par Dikmans avec le carolinensis. Il s'en distingue par des spicules à pointes simples et arrondies, un cône génital différent, des papilles zéro et sept allongées.

- Longistriata musculi Dikmans, 1935, parasite de Mus musculus et d'Oryzomys palustris, est placé dans le genre Hassalstrongylus.

- Hassalstrongylus musculi sensu Durette-Desset, 1972, parasite d'Oryzomys palustris, se différencie du musculi de Dikmans par un cône génital non hypertrophié, des spicules à pointes élargies mais symétriques. Cette nouvelle espèce est nommée: Hassalstrongylus forresteri n. sp.

2) Deux nouvelles espèces sont décrites :

- Boreostrongylus peromysci n. sp., parasite de différents Peromyscus, se distingue de $B$. carolinensis par un cône génital dépourvu d'expansion cuticulaire et des spicules à pointes simples, et de $B$. dikmansi par la pointe aiguë des spicules et le faible développement des arêtes latérales.

- Hassalstrongylus lichtenfelsi n. sp., parasite d'Oryzomys palustris, est très proche de $H$. musculi, les femelles étant identiques. Elle s'en distingue cependant par un fort développement du cône génital et des spicules à pointes aiguës. 
3) Connaissant huit espèces de Nippostrongylinae néarctiques sur les neuf actuellement nommées, nous proposons un tableau dichotomique de ces espèces.

\section{Summary.}

\section{On the nearctic Nippostrongylinae (Nematoda: Heligmosomidae).}

1) The author tries to explain the systematic position of four species ef nearctic Nippostrongylinae :

- Longistriata carolinensis Dikmans, 1935, parasite of Peromyscus maniculatus is placed in the genus Boreostrongylus.

- Dikmans had confused the Boreostrongylus dikmansi n. sp., parasite of Microtus ochrogaster with the carolinensis. It differs from it in spicules with simple and round tips, a distinct genital cone, and long-shaped papillae number zero and seven.

- Longistriata musculi Dikmans, 1935, parasite of Mus musculus and of Oryzomys palustris is placed in the genus Hassalstrongylus.

- Hassalstrongylus musculi sensu Durette-Desset, 1972, nec Dikmans, 1935, parasite of Oryzomys palustris, differs from Dikmans' musculi in an hypertrophied genital cone, spicules with enlarged points yet symmetrical. This new species is called : Hassalstrongylus forresteri $\mathrm{n}$. sp.

2) Two new species are described:

- Boreostrongylus peromysci n. sp., parasite of various Peromyscus, differs from $B$. carolinensis in a genital cone devoid of cuticular expansion and spicules with simple tips. It differs from $B$. dikmansi in the sharp-pointed spicules and the weak development of its lateral cuticular ridges.

- Hassalstrongylus lichtenfelsi n.sp., parasite of Oryzomys palustris, is very close to H. musculi, their females being identical. However it differs from it in a great development of the genital cone and sharp-pointed spicules.

3) As the author knows eight species of nearctic Nippostrongylinae out of the nine named at present, she proposes a dichotomic table of these species.

Les Nippostrongylinae néarctiques appartiennent uniquement à deux genres : Boreostrongylus et Hassalstrongylus, qui phylétiquement dérivent l'un de l'autre. Dans chaque genre, les espèces sont morphologiquement proches les unes des autres, et une certaine confusion s'était glissée dans les travaux de Dikmans (1935) et les nôtres (Durette-Desset, 1972).

L'envoi de spécimens de Dikmans et de parasites néarctiques, dû à l'obligeance de nos collègues américains les $\mathrm{D}^{r s} \mathrm{R}$. Lichtenfels et M. J. Kinsella, que nous remercions vivement, nous a permis de clarifier la position systématique de ces espèces et de proposer un tableau dichotomique des Nippostrongylinae néarctiques.

Les spécimens ont été déposés soit dans les collections Helminthologiques de l'United States National Museum, soit dans celles du Museum de Paris. 

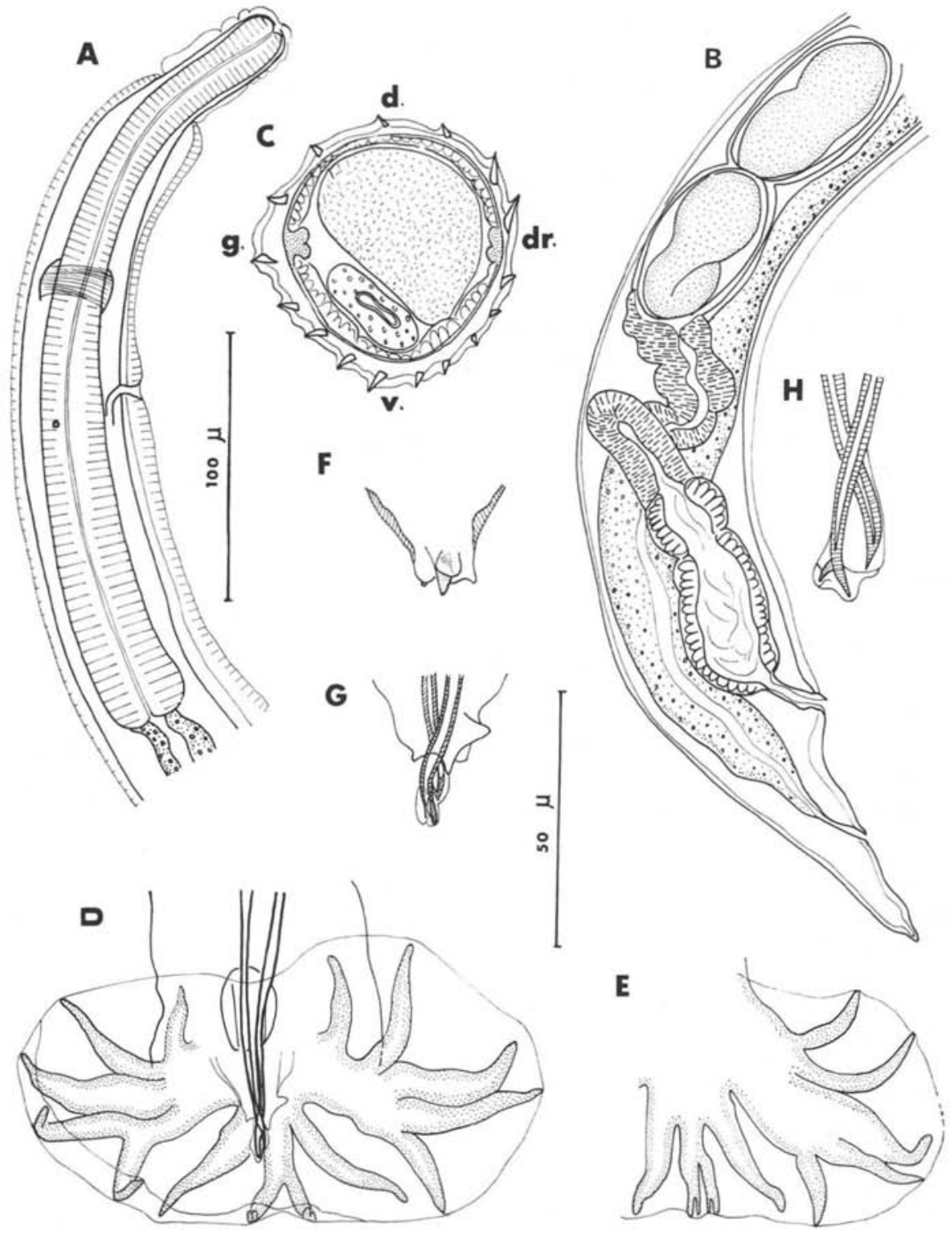

Fig. 1. - Boreostrongylus carolinensis (Dikmans, 1935). n.cb. A) ᄋ, extrémité antérieure, vue latérale droite, B) $\circ$, extrémité postérieure, vue latérale droite; C) $ᄋ$, coupe transversale au milieu du corps. D) $\delta$, bourse caudale, vue ventrale; E) autre o', bourse caudale, lobe gauche étalé, vue ventrale; F) $\hat{\delta}$, cône génital disséqué, vue ventrale; $\mathrm{G}) \hat{o}$, cône génital en place, vue sub-latérale; $\mathrm{H})$ ô, extrémité postérieure des spicules disséqués. A, $\mathrm{B}, \mathrm{D}, \mathrm{E}, e ́ c h .100 \mu ; \mathrm{C}, \mathrm{F}, \mathrm{G}, \mathrm{H}$, éch. $50 \mu$. 


\section{I. - DETERMINATION DES ESPECES}

\section{Boreostrongylus carolinensis}

(Dikmans, 1935) n. comb.

\section{$=$ Longistriata carolinensis}

Dikmans, 1935

MATÉRIEL de RÉFÉRENCE : 1 o 0,1 \%, $\mathrm{n}^{\circ} 328$.

Hôte : Peromyscus maniculatus Wagner.

Origine géographiQue: Great Smokey Mountains, North Carolina.

LOCALISATION : Intestin.

Autre matériel: 1) quelques $\delta$ ' et $q$ provenant du lot précédent; 2) quelques $\delta$ chez le même hôte et dans la même région, $\mathrm{n}^{\circ} 303$; U.S.N.M. Helm. coll. $n^{\circ} 61898$ (2 tubes).

\section{Redescription.}

Petits Nématodes enroulés sur plusieurs tours de spire le long de leur ligne ventrale. Pore excréteur et deirides situés un peu en arrière de l'anneau nerveux.

SYNLOPHE: Chez les deux sexes, le corps est parcouru longitudinalement par $16(\hat{o})$, 17 (\%) arêtes cuticulaires qui débutent sur le bord postérieur de la vésicule céphalique et s'étendent jusqu'à environ $135 \mu$ en avant de la bourse caudale chez le $\hat{\delta}$; jusqu'au niveau du sphincter chez la 우. La disposition des arêtes et les gradients de taille sont de type Boreostrongylus (fig. 1, C).

MÂLE : Corps long de $2,45 \mathrm{~mm}$, large de $55 \mu$ dans sa partie moyenne. Vésicule céphalique haute de $43 \mu \times 22 \mu$ de large. Anneau nerveux, pore excréteur et deirides situés respectivement à $120 \mu, 170 \mu$ et $180 \mu$ de l'apex ; œsophage long de $285 \mu$.

Contrairement à la description de Dikmans, la bourse caudale est sub-symétrique avec un lobe gauche un peu plus développé (1), mais la disposition des côtes bursales est la même que dans la description originale (fig. 1, D).

Spicules sub-égaux, longs de $430 \mu$. Le spicule gauche est bifurqué en deux branches inégales à son extrémité distale. Les pointes des spicules sont enfermées dans une membrane commune (fig. 1, H).

Gubernaculum haut de $22 \mu$ sur $20 \mu$ de large. Cône génital portant sur sa face ventrale une expansion de forme triangulaire en vue latérale. Sur l'animal, en vue ventrale, cette expansion est rejetée soit vers la droite, soit vers la gauche et le cône génital se trouve en vue sub-latérale. La papille zéro est pointue, les papilles 7 peu développées, de forme arrondie (fig. 1, F, G).

(1) Nous n'avons pas étalé complètement le mâle utilisé comme matériel de référence, par crainte de l'abîmer. Seuls le lobe dorsal et le lobe gauche de ce mâle sont figurés (fig. 1, E). Nous donnons également une figure d'un autre mâle, incomplètement étalée, mais permettant de situer la position des côtes bursales (fig. 1, D). 
Femelle : Corps long de $3,8 \mathrm{~mm}$, large de $70 \mu$ dans sa partie moyenne. Vésicule céphalique haute de $48 \mu$ sur $24 \mu$ de large. Anneau nerveux, pore excréteur et deirides situés respectivement à $120 \mu, 170 \mu$ et $180 \mu$ de l'apex. Oesophage long de $295 \mu$ (fig. 1, A).

Monodelphie. La vulve s'ouvre à $97 \mu$ de la pointe caudale. Vagina vera : $22 \mu$; vestibule, sphincter et trompe longs respectivement de $57 \mu, 23 \mu$ et $120 \mu$. L'utérus mesure $520 \mu$ et contient huit œufs à différents degrés de segmentation, hauts de $65 \mu \times 35 \mu$ (fig. 1, B).

Queue longue de $41 \mu$, à extrémité caudale triangulaire (fig. 1, B).

\section{Discussion.}

En recherchant le matériel type de Longistriata carolinensis Dikmans, 1935, parasite de Peromyscus maniculatus et originaire des Great Smokey Mountains, le $\mathrm{D}^{r}$ Lichtenfelds a constaté qu'il n'existait plus que les femelles. Le $\mathrm{D}^{r}$ Lichtenfelds a eu alors l'obligeance de nous adresser :

1) un lot de la même espèce et de la même région que les spécimens types ;

2) un lot parasite de Microtus ochrogaster que Dickmans avait identifié à l'espèce carolinensis.

A l'examen, nous constatons qu'il s'agit de deux espèces différentes, mais nous n'avons aucune hésitation à identifier au carolinensis le matériel provenant de Peromyscus (étudié ci-dessus), car la pointe des spicules (fig. 1, H) correspond à la description de Dikmans (spicules « expanded»), alors que les parasites de Microtus ne possèdent pas ce caractère (fig. $2, \mathrm{E}$ ). Ces derniers seront décrits ci-après sous le nom de Boreostrongylus dikmansi n. sp.

D'après nos conceptions (cf. Durette-Desset, 1971), nous classons l'espèce de Dikmans dans le genre Boreostrongylus dont elle présente les principaux caractères, et en particulier le synlophe.

\section{Boreostrongylus dikmansi n. sp.}

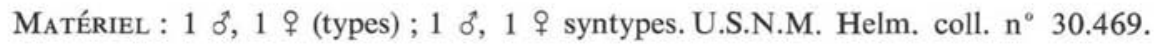

HôTE : Microtus ochrogaster Wagner.

Localisation : Intestin.

ORIGINE GÉOGRAPHIQUE : Vincennes, Indiana.

\section{Description.}

Petits Nématodes plus ou moins enroulés le long de leur ligne ventrale. Pore excréteur et deirides situées un peu en arrière de l'anneau nerveux. Glandes excrétrices bien visibles. 


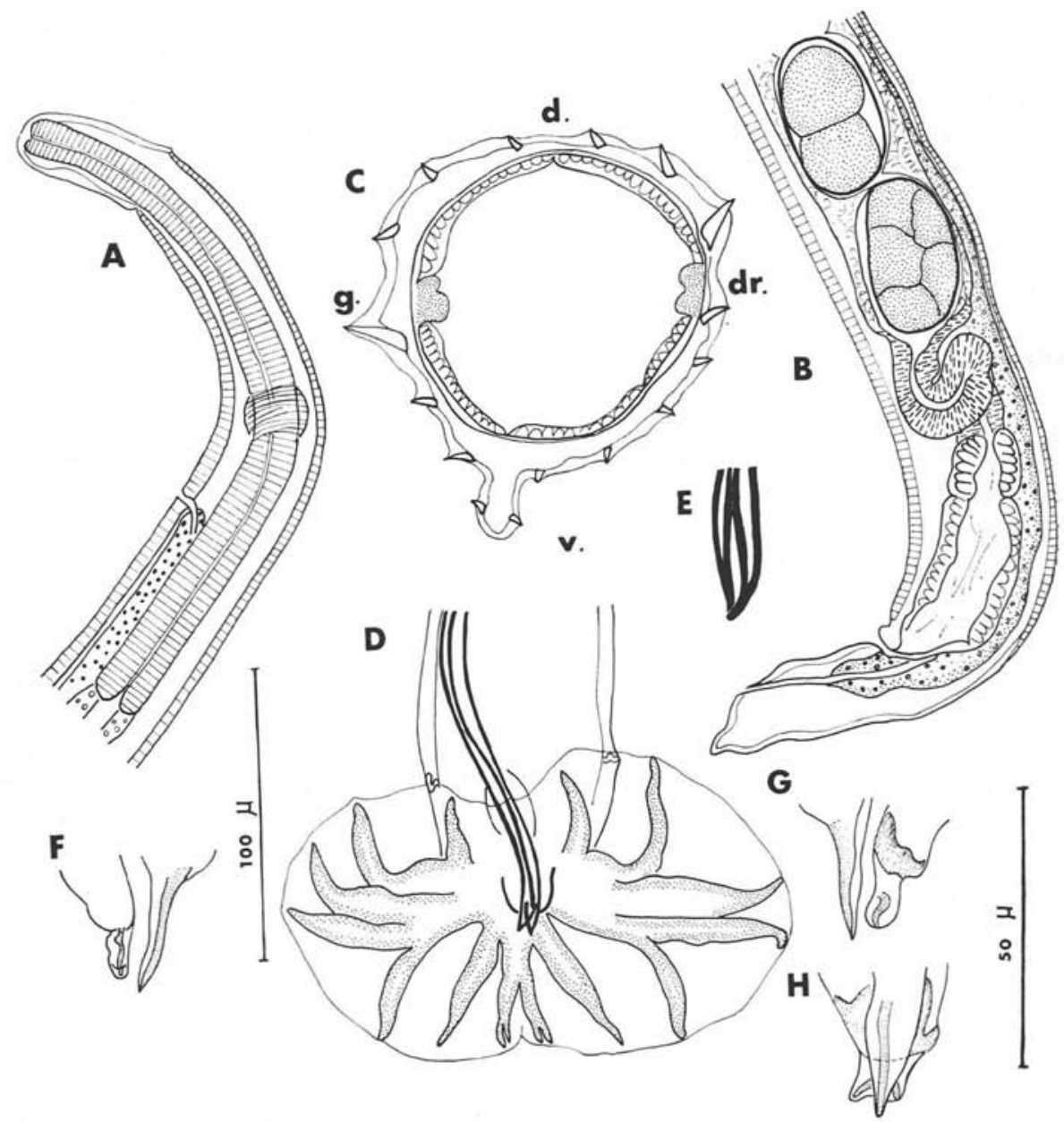

FIG. 2. - Boreostrongylus dikmansi n.sp.; A) ९, extrémité antérieure, vue latérale gauche ; B) + , extrémité postérieure, vue latérale gauche; C) + , coupe transversale au milieu du corps; D) $\hat{\delta}$, bourse caudale, vue ventrale; E) $\hat{\delta}$, pointe des spicules disséqués; F) $\hat{\jmath}$, cône génital, vue latérale droite; $\mathrm{G})$ id., vue latérale gauche; $\mathrm{H})$, id., vue ventrale. $\mathrm{A}, \mathrm{B}, \mathrm{D}$, éch. $100 \mu ; \mathrm{C}, \mathrm{E}, \mathrm{F}, \mathrm{G}, \mathrm{H}$, éch. $50 \mu$.

Synlophe: Par manque de matériel, nous n'avons étudié le synlophe que chez la . Le corps est parcouru longitudinalement par seize arêtes cuticulaires qui débutent sur le bord postérieur de la vésicule céphalique et s'étendent jusqu'au niveau de la vulve. La disposition des arêtes et les gradiens de taille sont de type Boreostrongylus (fig. 2, C).

MÂle: (holotype): Corps long de $1,7 \mathrm{~mm}$, large de $60 \mu$ dans sa partie moyenne. Vésicule céphalique haute de $42 \mu$ sur $20 \mu$ de large. Anneau nerveux, pore excréteur et deirides situés respectivement à $110 \mu, 155 \mu$ et $155 \mu$ de l'apex. Oesophage long de $255 \mu$. 
Bourse caudale sub-symétrique avec un lobe gauche un peu plus développé, haute de $80 \mu$ sur $180 \mu$ de large. Côtes 8 naissant à la racine de la côte 9 . Cette dernière est divisée en deux rameaux à la moitié de sa hauteur. Autres côtes figurées en 2, D.

Spicules sub-égaux, ailés, longs de $370 \mu$. Leur extrémité distale est arrondie (fig. 2, E). Gubernaculum haut de $25 \mu$ sur $14 \mu$ de large. Cône génital bien développé haut de $28 \mu$. La papille zéro est de forme triangulaire, les papilles 7 se présentent comme de fines languettes et sont enfermées dans une membrane commune (fig. 2, F, G, H).

Femelle (allotype): Corps long de $2,1 \mathrm{~mm}$, large de $60 \mu$ dans sa partie moyenne. Vésicule céphalique haute de $47 \mu$ sur $22 \mu$ de large. Anneau nerveux, pore excréteur et deirides situés respectivement à $130 \mu, 195 \mu$ et $195 \mu$ de l'apex. Oesophage long de $255 \mu$ (fig. 2, A).

Monodelphie. La vulve s'ouvre à $73 \mu$ de l'extrémité caudale. Vagina vera: $15 \mu$; vestibule, sphincter et trompe longs respectivement de $46 \mu, 22 \mu$ et $90 \mu$. Utérus long de $400 \mu$. Il contient six œufs segmentés hauts de $52 \mu$ sur $32 \mu$ de large.

Queue longue de $23 \mu$, assez trapue mais à extrémité pointue (fig. 2, B).

\section{Discussion.}

Comme nous l'avons vu plus haut. les spécimens du Microtus ne sont pas identifiables au carolinensis de Dikmans, car la pointe des spicules n'est pas complexe (fig. 2, E). De plus, ils en diffèrent par la forme du cône génital, l'allongement de la papille zéro et des papilles 7. Enfin, l'arête latérale gauche est beaucoup plus développée et la queue de la femelle plus courte.

Nous rangeons ces spécimens dans le genre Bereostrongylus dont ils présentent les principaux caractères, et nous les nommons Boreostrongylus dikmansi n. sp.

\section{Boreostrongylus peromysci n. sp.}

1) Matériel type: $5 \delta, 2$ (176 MA).

Hôte : Peromyscus floridanus Chapman.

LOCALISATION : Intestin grêle.

ORIGINE GÉOGRAPHIQUE: Highlands Country Florida.

2) Autre matériel: Dans l'intestin grêle de deux $P$. gossypinus Le Conte originaires soit de la même région (200 MA), soit de Cedar Key, Levy Country Florida (229 Ka).

\section{Description.}

Petits Nématodes enroulés le long de leur ligne ventrale. L'enroulement affecte surtout la première moitié du corps qui comprend deux à trois tours de spire. La 


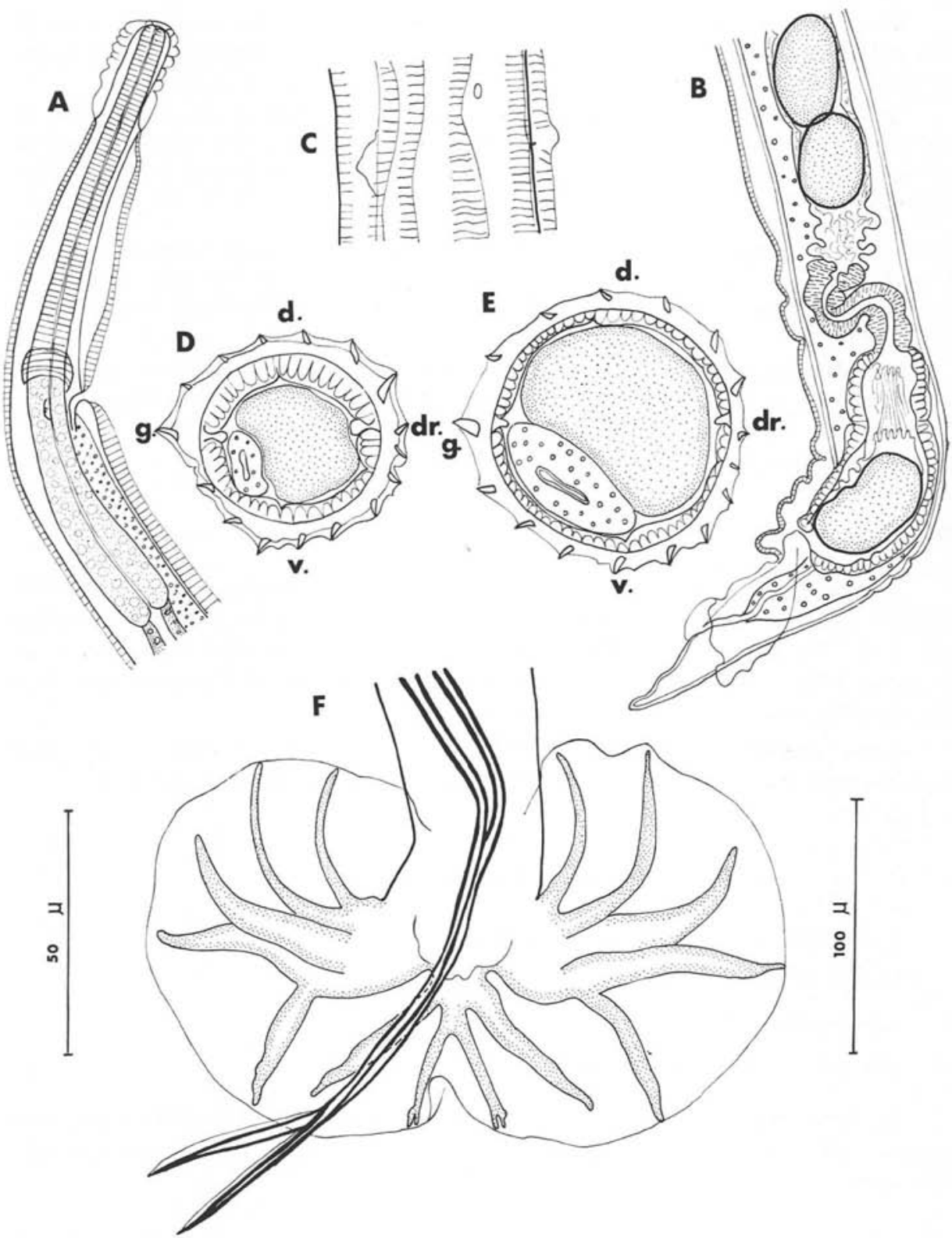

FIG. 3. - Boreostrongylus peromysci n.sp.; A) \&, extrémité antérieure, vue latérale droite; B) $q$, extrémité postérieure, vue latérale gauche; C) $\hat{\delta}$, pore excréteur et deirides, vue ventrale; D) $\hat{\delta}$, coupe transversale au milieu du corps; E) $ᄋ$, id.; F) $\hat{\delta}$, bourse caudale. vue ventrale. $\mathrm{A}, \mathrm{B}, \mathrm{F}$, éch. $100 \mu ; \mathrm{C}, \mathrm{D}, \mathrm{E}$, éch. $50 \mu$. 
partie postérieure est déroulée. Chez les deux sexes, l'extrémité postérieure du corps est coudée, formant un angle d'environ $45^{\circ}$ chez le $\delta, 60^{\circ}$ chez la + . Pore excréteur situé juste en arrière de l'anneau nerveux. Deirides postérieures à celui-ci (fig. 3, C).

SYNLOPHE: Chez les deux sexes, le corps est parcouru longitudinalement par seize arêtes cuticulaires dans sa partie moyenne (fig. 3, D, E). Les arêtes naissent à différents niveaux en arrière de la vésicule céphalique et s'étendent jusqu'au niveau de la bourse caudale chez le $\hat{\delta}$, et de la vulve chez la ${ }^{\circ}$. Dans le tiers antérieur du corps, les arêtes ventrales gauches forment des comarêtes et le nombre d'arêtes n'est que de quatorze. Excepté à ce niveau, les arêtes latérales droite et gauche sont plus développées que les autres. La pointe des arêtes est dirigée de la ligne droite, ventrale-droite vers la ligne gauche pour les deux faces.

MÂLE : Corps long de $2,4 \mathrm{~mm}$, large de $90 \mu$ dans sa partie moyenne. Vésicule céphalique haute de $42 \mu \times 23 \mu$ de large. Anneau nerveux, pore excréteur et deirides situés respectivement à $135 \mu, 175 \mu$ et $190 \mu$ de l'apex. Oesophage long de $265 \mu$.

Bourse caudale sub-symétrique. Côtes 2 et 3 plus longues que les côtes 4 . Côtes 4 et 5 divergentes à leur extrémité. Côtes 8 naissant à la racine de la côte dorsale et légèrement plus courtes que cette dernière. Côte dorsale profondément divisée en deux rameaux euxmêmes bifurqués (fig. 3, F).

Spicules, fins, ailés, subégaux, longs de $300 \mu$, à extrémité aiguë (fig. 3, F). Gubernaculum non observé. Cône génital peu marqué. Papilles 7 arrondies, papille zéro non vue.

Femelle: Corps long de $2,8 \mathrm{~mm}$, large de $50 \mu$ dans sa partie moyenne. Vésicule céphalique haute de $42 \mu \times 22 \mu$ de large. Anneau nerveux, pore excréteur et deirides situés respectivement à $135 \mu, 150 \mu$ et $155 \mu$ de l'apex. Oesophage long de $250 \mu$ (fig. 3, A).

Monodelphie. La vulve s'ouvre à $100 \mu$ de l'extrémité caudale. Vagina vera très court Ovéjecteur long de $162 \mu$ (vestibule $62 \mu$; sphincter $30 \mu$; trompe $70 \mu$ ). L'utérus long dı $330 \mu$ contient huit œufs non embryonnés hauts de $42 \mu \times 28 \mu$ de large.

Queue longue de $42 \mu$, arrondie à son extrémité. Elle peut s'invaginer à l'intérieur dt la cuticule (fig. 3, B).

\section{Discussion.}

Les spécimens du Peromyscus floridanus peuvent être rangés dans le genre Boreostrongylus dont ils présentent les principaux caractères.

Ce genre est représenté actuellement par six espèces, toutes holarctiques. Nos spécimens sont très proches de $B$. carolinensis et de $B$. dikmansi, car la disposition des côtes bursales et le synlophe sont pratiquement identiques. Cependant, les spécimens du Peromyscus se différencient: 1) des deux espèces ci-dessus par des ventroventrales plus longues et la possibilité d'invagination de la queue de la $q$; 2) de $B$. carolinensis par un cône génital dépourvu d'expansion cuticulaire ventrale et des spicules à pointe simple ; 3 ) de $B$. dikmansi par le faible développement des arêtes latérales et par des spicules à extrémité pointue et non arrondie.

Nous pensons donc que cette espèce est nouvelle et nous proposons de la nommer Boreostrongylus peromysci n. sp. 


\section{Hassalstrongylus musculi}

(Dikmans, 1935) n. cb. sensu Dikmans, 1935, nec Durette-Desset, 1972

MAtÉRIEL : 5 б, 5 q syntypes, U.S.N.M. Helm. coll. $\mathrm{n}^{\circ} 30456$.

Hôte: Mus musculus L.

LOCALISATION : Intestin grêle.

ORIGINE GÉOGRAPHIQUE : Jeanerette, Louisiane.

Autre matériel : $7 \delta^{\circ}$, coparasites d'Hassalstrongylus lichtenfelsi $\mathrm{n}$. sp.

Hôte: Oryzomys palustris Harlan $(228 \mathrm{Ka})$.

LOCALISATION : Intestin grêle.

Origine GÉographiQue: Paynes Prairies, Alachua Country, Florida.

\section{Description.}

Petits Nématodes enroulés très lâchement le long de leur ligne ventrale. Les ơ sont le plus souvent complètement déroulés. Pore excréteur situé entre l'anneau nerveux et la fin de l'œsophage à des niveaux variables. Deirides un peu postérieures, très discrètes. Présence d'une dilatation cuticulaire ventrale.

SYNLOPHE: Le corps est parcouru par 23 à 25 arêtes cuticulaires dans sa partie moyenne. Les arêtes naissent à différents niveaux sur la ligne latérale gauche (fig. 5, A), en arrière de la vésicule céphalique sur la face latérale droite. Elles se poursuivent jusqu'au niveau de la bourse caudale chez le $\hat{\delta}$, jusqu'au niveau de l'anus chez la $\uparrow$. Les arêtes ventrales et dorsales sont les plus développées (fig. 5, C, D).

MÂLE: Corps long de $2,7 \mathrm{~mm}$; large de $110 \mu$ dans sa partie moyenne, dont $60 \mu$ seulement pour le corps proprement dit. Anneau nerveux, pore excréteur et deirides situés respectivement à $120 \mu, 155 \mu$ et $160 \mu$ de l'apex. Oesophage mesurant $270 \mu(120 \mu$ oesophage musculaire; $140 \mu$ œsophage glandulaire) (fig. 5, B). Bourse caudale sub-symétrique, presqu'aussi haute que large. Côtes figurées en $5, \mathrm{~F}$. Côtes 8 naissant à la racine de la côte dorsale, épaisses. Côte 9 divisée en deux rameaux dans son tiers postérieur (fig. 5, I).

Spicules sub-égaux, longs de $390 \mu$, assez complexes. Leur pointe distale est élargie, mais plus fortement chez le spicule droit où elle prend la forme d'un pied (fig. $5, \mathrm{G}, \mathrm{H}$ ). Gubernaculum non observé. Cône génital hypertrophié, de forme triangulaire, haut de $62 \mu$. Papille zéro fine et allongée. Papilles 7 dédoublées, disposées en éventail (fig. 5, J, K).

Femelle (2): Corps long de $2,35 \mathrm{~mm}$, large de $75 \mu$ dans sa partie moyenne. Vésicule céphalique haute de $45 \mu$ sur $20 \mu$ de large. Anneau nerveux, pore excréteur, et deirides situés respectivement à $110 \mu, 160 \mu$ et $165 \mu$ de l'apex. Oesophage long de $270 \mu$.

(2) Le tube $228 \mathrm{Ka}$ contenait 31 \$, 7 वे d'Hassalstrongylus musculi et 8 वे d'H. lichtenfelsi n. sp. décrits ci-après. Nous n’avons trouvé aucun élément morphologique nous permettant de séparer les femelles et de les rapporter à une des deux espèces. 


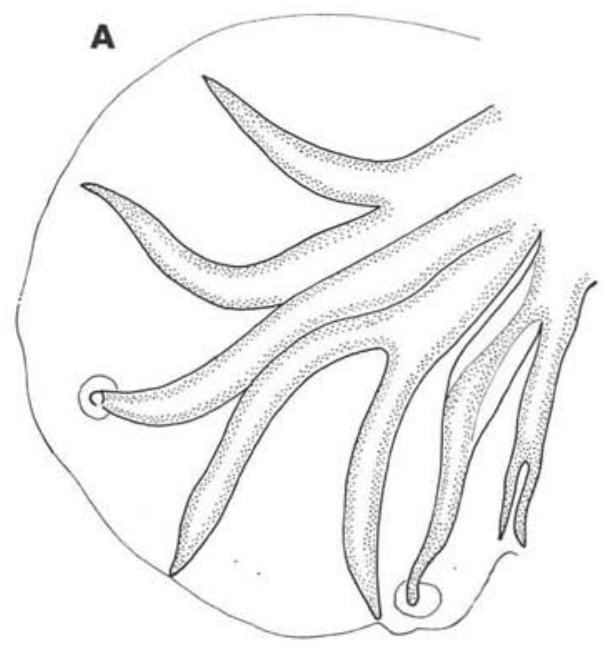

B

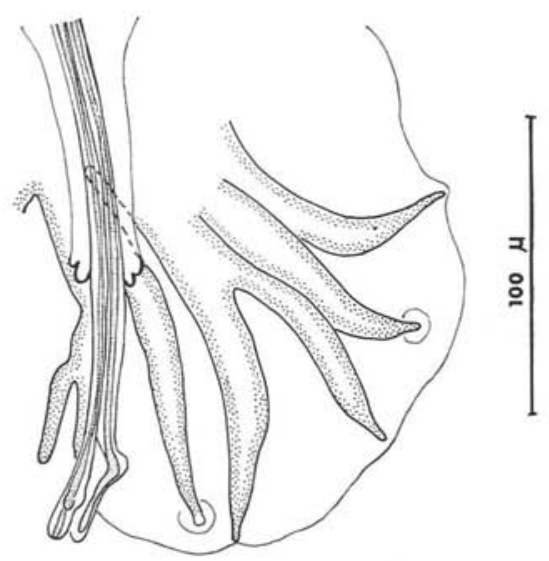

FIG. 4. - Hassalstrongylus musculi [(Dikmans, 1935) nec Durette-Desset, 1972] n.cb. Syntype ơ chez Mus musculus. A) lobe gauche de la bourse caudale; B) lobe droit.

Monodelphie. La vulve s'ouvre à $72 \mu$ de l'extrémité caudale. Vagina vera: $25 \mu$. Vestibule, sphincter et trompe longs respectivement de $53 \mu, 25 \mu$ et $60 \mu$. L'utérus mesure $270 \mu$ et contient 4 œufs non embryonnés, hauts de $55 \mu$ sur $23 \mu$ de large (fig. 5, E).

Queue pointue, longue de $38 \mu$ (fig. 5, E).

\section{Discussion.}

Nous n'avons constaté aucune différence morphologique entre les Nématodes provenant de Mus musculus et ceux parasites de l'Oryzomys palustris. Pour ne pas abîmer les spécimens types qui paraissent très fragiles, nous avons choisi de faire la redescription sur le matériel de l'Oryzomys, et nous donnons seulement une figure d'un ơ syntype servant de comparaison (fig. 4, A, B).

En 1972, nous avons par erreur identifié un parasite d'Oryzomys palustris de Floride comme étant le musculi de Dikmans, et nous avions rangé l'espèce dans le genre Hassalstrongylus. Nous proposons pour cette nouvelle espèce le nom de Hassalstrongylus forresteri n. sp. (= Hassalstrongylus musculi Durette-Desset, 1972). Elle se différencie du musculi de Dikmans : 1) par un cône génital non hypertrophié ; 2) par des spicules à pointes élargies, mais de façon identique chez les deux spicules, alors que chez le musculi l'extrémité postérieure du spicule droit est plus fortement développée que celle du spicule gauche ; 3) par des spicules plus courts.

La position systématique du musculi de Dikmans nous paraît intéressante du point de vue phylétique. En effet, l'espèce présente à la fois des caractères des genres Hassalstrongylus et Stilestrongylus qui à notre avis (cf. Durette-Desset, 1971) appartiennent à la même lignée. 


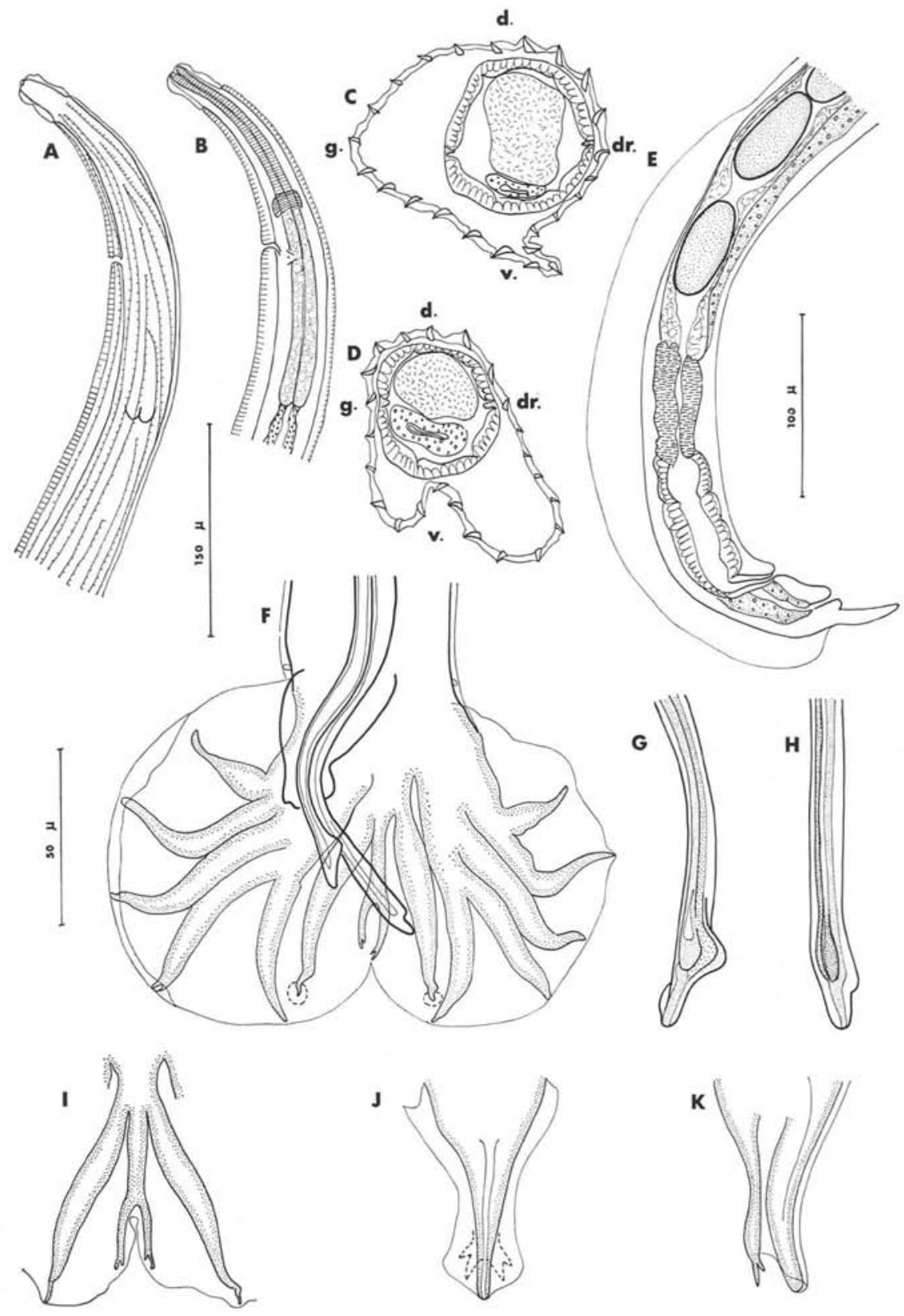

Fig. 5. - Hassalstrongylus musculi [(Dikmans, 1935) nec Durette-Desset, 1972] n.cb. chez Oryzomys palustris. A) $\hat{\delta}$, extrémité antérieure, naissance des arêtes cuticulaires, vue latérale gauche; $\mathrm{B}) \hat{\delta}$, extrémité antérieure, vue latérale gauche; C) $\hat{\delta}$, coupe transversale au milieu du corps; D) + , id. ; E) $\uparrow$, extrémité postérieure, vue latérale droite; F) $\hat{\delta}$, bourse

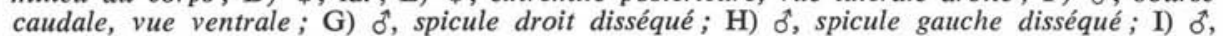
côtes 9 et 8 , vue dorsale; J, K) $\delta$, cône génital, successivement, vue ventrale et latérale droite. A, B, éch. $150 \mu ; \mathrm{C}, \mathrm{D}, \mathrm{J}, \mathrm{K}, \mathrm{G}, \mathrm{H}$, éch. $50 \mu ; \mathrm{E}, \mathrm{F}, \mathrm{I}, e ́ c h .100 \mu$. 
L'évolution de cette lignée se serait faite en Amérique du Nord, du nord au sud, selon les éléments suivants: 1) augmentation du nombre des arêtes cuticulaires ; 2) changement de l'axe d'orientation de ces arêtes ; 3) allongement du cône génital.

Nous avions divisé cette lignée en trois genres: Boreostrongylus, Hassalstrongylus et Stilestrongylus, le premier étant typique de la région paléarctique, le second de la région néarctique et le dernier de la région néo-tropicale.

Le phénomène évolutif étant progressif, les coupures génériques sont nécessairement arbitraires et les localisations géographiques se recoupent largement en Amérique (Boreostrongylus déborde sur l'Amérique du Nord et Hassalstrongylus sur l'Amérique du Sud).

L'espèce de Dikmans constitue précisément une transition entre les genres Hassalstrongylus et Stilestrongylus.

1) Le synlophe est de type Hassalstrongylus évolué: le nombre d'arêtes est élevé (23), et alors que dans le genre Hassalstrongylus les arêtes gauches, ventrales et dorsales sont plus développées que les autres arêtes, ici seules les ventrales et les dorsales sont les plus fortes. Du point de vue évolutif, ceci nous paraît une étape vers l'égalisation de la taille des arêtes, ce qui sera réalisé dans le genre Stilestrongylus.

2) Le cône génital est de type Stilestrongylus, c'est-à-dire hypertrophié.

Il ne nous paraît pas nécessaire de créer un genre nouveau pour cette espèce et nous choisissons de la ranger dans le genre Hassalstrongylus en attribuant une importance prépondérante aux caractères fournis par le synlophe.

\section{Hassalstrongylus licbtenfelsi n. sp.}

MATÉRIEL TYPE : 8 o coparasites d'Hassalstrongylus musculi.

Hôte : Oryzomys palustris Harlan (228 Ka).

LOCALISATION : Intestin grêle.

Origine géographiQue: Paynes Prairie, Alachua Country, Florida.

\section{Description.}

Petits Nématodes enroulés selon une spire sénestre le long de leur ligne ventrale. Cette spire est plus ou moins lâche selon les spécimens. Elle comporte généralement un seul tour.

Pore excréteur situé à mi-hauteur entre l'anneau nerveux et la fin de l'œsophage. Deirides au même niveau, petites, en forme de bâtonnets. Séparation entre l'œsophage musculaire et l'œsophage glandulaire nette.

SyNLOPHE: Il est identique à celui de l'espèce précédente, avec cependant 25 arêtes au lieu de 23 (fig. 6, B). 
Mensurations: Corps long de $2,1 \mathrm{~mm}$, large de $65 \mu$ dans sa partie moyenne. Vésicule céphalique haute de $40 \mu$ sur $22 \mu$ de large. Anneau nerveux, pore excréteur et deirides situés respectivement à $115 \mu, 170 \mu$ et $175 \mu$ de l'apex. Oesophage long de $240 \mu(105 \mu$ oesophage musculaire; $135 \mu$ oesophage glandulaire) (fig. 6, A).
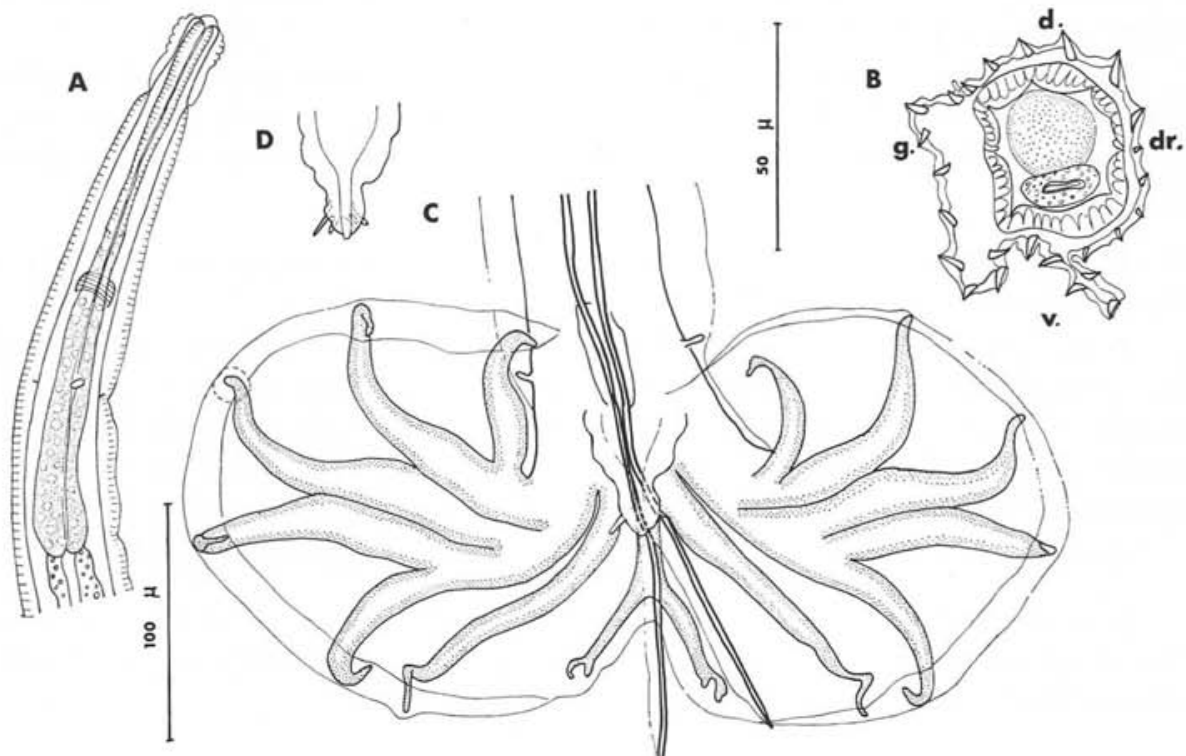

Fig. 6. - Hassalstrongylus lichtenfelsi n.sp.; A) ô, extrémité antérieure, vue ventrale; B) $\hat{\delta}$, coupe transversale au milieu du corps; C) $\hat{\delta}$, bourse caudale, vue ventrale; D) $\hat{o}$, détail du cône génital avec la papille zéro et les papilles 7 dédoublées, vue ventrale. $\mathrm{A}$, éch. $100 \mu ; \mathrm{B}, \mathrm{C}, \mathrm{D}$, éch. $50 \mu$.

Bourse caudale sub-symétrique. Papilles prébursales non observées. Côtes 8 naissant à la racine de la côte dorsale. Cette dernière est profondément divisée en deux branches elles-mêmes bifurquées à leur extrémité (fig. 6, C).

Spicules fins, ailés, sub-égaux, longs de $185 \mu$. Leur extrémité postérieure est aiguë. Ils glissent dans un gubernaculum haut de $25 \mu$ sur $10 \mu$ de large, de forme rectangulaire.

Cône génital bien développé. Papille zéro longue et fine. Papilles 7 dédoublées en 2 fines languettes (fig. 6, D).

\section{Discussion.}

Cette espèce est très proche de $H$. musculi aussi bien par la disposition des côtes bursales que par le synlophe. De plus, les femelles sont pratiquement identiques. Elle se distingue cependant de $H$. musculi par deux éléments constants et associés : un cône 
génital allongé mais deux fois plus petit et des spicules à extrémité pointue et non élargie.

Nous considérons donc cette espèce comme nouvelle et nous proposons de la nommer Hassalstrongylus lichtenfelsi n. sp.

\section{II. - TABLEAU DICHOTOMIQUE DES NIPPOSTRONGYLINAE NEARCTIQUES}

Ayant eu l'occasion d'étudier huit espèces de Nippostrongylinae sur les neuf actuellement nommées en Amérique du Nord, nous pensons qu'il est possible de les différencier de la façon suivante :

1 - (8) Nombre d'arêtes cuticulaires inférieur à $19 \ldots \ldots \ldots \ldots \ldots$ Boreostrongylus

2 - (7) Spicules à pointe simple, aiguë ou arrondie.

3 - (4) Nombre d'arêtes inférieur à 16 ; côtes 8 naissant au $1 / 3$ de la hauteur de la côte 9 . Arêtes latérales fortes, spicules à extrémité pointue.

Parasites de Neofiber alleni ............... k. kinsellai (Durette-Desset, 1969)

4 - (3) Nombre d'arêtes égal à 16 : côtes 8 naissant à la racine de la côte 9.

5 - (6) Arêtes latérales fortes, gradients de taille des arêtes bien marqués, spicules à pointe arrondie.

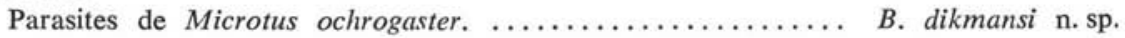

6 - (5) Arêtes latérales peu développées, gradients de taille des arêtes mal définis, spicules à pointe aiguë.

Parasites de Peromyscus floridanus et $P$. gossypinus ........ B. peromysci n. sp.

7 - (2) Spicules à pointe complexe.

Côtes 8 naissant à la racine de la côte 9, Cône génital avec expansion cuticulaire ventrale, arêtes latérales peu développées. Parasites de Peromyscus maniculatus ... $\ldots \ldots \ldots \ldots \ldots \ldots \ldots \ldots \ldots \ldots \ldots \ldots \ldots \ldots$ B. carolinensis (Dikmans, 1935)

8 - (1) Nombre d'arêtes cuticulaires supérieur à $19 . \ldots \ldots \ldots \ldots \ldots$ Hassalstrongylus

9 - (14) Cône génital non hypertrophié.

10 - (13) Nombre d'arêtes égal à 23.

11 - (12) Côte 9 profondément divisée, spicules à pointe simple.

Parasites de Sigmodon hispidus ............... H. aduncus (Chandler, 1932)

12 - (11) Côte 9 divisée aux $2 / 3$ de sa hauteur, spicules élargis à leur extrémité distale.

Parasites d'Oryzomys palustris $\ldots \ldots \ldots \ldots \ldots \ldots \ldots \ldots \ldots \ldots \ldots \ldots \ldots \ldots \ldots \ldots$ forresteri $\mathrm{n} . \mathrm{sp}$.

13 - (10) Nombre d'arêtes supérieur à 23.

Spicules à extrémité pointue, côte 9 divisée à la moitié de sa hauteur. Parasites

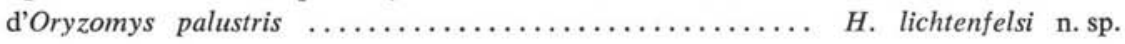

14 - (9) Cône génital hypertrophié.

Spicules à pointe fortement élargie et asymétrique. Parasites de Mus musculus et Oryzomys palustris ...................... H. musculi (Dikmans, 1935) 
Nous n'avons pas inclus $B$. dalrymplei Dikmans, 1935, parasite d'Ondatra zibethica et Microtus pennsylvanicus dans ce tableau dichotomique, car nous n'en connaissons pas le synlophe.

Cette espèce est très proche de $B$. kinsellai (disposition des côtes bursales semblables, côtes 8 grêles et naissant au tiers de la hauteur de la côte 9). Elle s'en distingue par un cône génital plus long, l'absence de macules sur la cuticule bursale, des spicules et un ovéjecteur plus longs.

\section{Bibliographie}

Chander (A. C.), 1932. - A new species of Longistriata (Nematoda) from the cotton Rat, Sigmodon hispidus, with notes on the division of Heligmosominae into genera. J. Parasit., 19 (1), 25-30.

Dikmans (G.), 1935. - New Nematodes of the genus Longistriata in rodents. J. Wash. Ac. Sc., $25,78-81$.

DuRette-Desset (M. C.), 1969. - Etude du système des arêtes cuticulaires de trois Nématodes Héligmosomes : Longistriata kinsellai n. sp., L. seurati Travassos et Darriba, 1929, L. hokkaidensis Chabaud, Rausch et Desset, 1963, parasites de Rongeurs. Ann. Parasit. hum. comp., 44 (5), 617-624.

—, 1971. - Essai de classification des Nématodes Héligmosomes. Corrélations avec la paléobiogéographie des hôtes. Mém. Mus. Nat. Hist. Nat., nouv, sér., sér. A. Zool,, 49, 126 p.

—, 1972. — Compléments morphologiques à l'étude de quelques Nématodes Héligmosomes, parasites de Rongeurs américains. Ann. Parasit., hum. comp. 47 (2), 243-249. 